DNA INTERFERENCE

Old Ago, new trick

Nature doi:10.1038/nature12971

In eukaryotes, Argonaute (Ago) proteins are the 'slicers' of RNA interference (RNAi) pathways and are guided to their RNA targets by complementary small RNAs anchored in the Ago pocket. Ago proteins are also found in prokaryotes, but their functional roles remain puzzling, as archaea and bacteria lack RNAi machinery. A study in 2013 provided evidence that Ago from the bacterium Rhodobacter sphaeroides uses small RNAs and DNAs to target invading plasmid DNA. Swarts et al. now demonstrate that Thermus thermophilus Ago (TtAgo) mediates DNA-guided cleavage of plasmid DNA. The authors found that T. thermophilus strains lacking functional TtAgo exhibit higher transformation efficiencies and plasmid levels than wild-type strains, suggesting that TtAgo regulates uptake or processing of foreign plasmid DNA. TtAgo co-purifies with 5'-phosphorylated DNAs ( 13-25 nucleotides in size), which the authors refer to as 'small interfering DNA' (siDNA). Further analyses revealed that TtAgo-siDNA complexes can cleave complementary single-stranded DNA under in vitro conditions, and TtAgo, complexed with siDNAs derived from invading plasmid sequences, can selectively nick negatively supercoiled plasmids or linearize them if a pair of nicking complexes with nearby targets is used. Though the process by which siDNAs are generated from plasmid DNA remains unknown, the authors propose that DNA-guided DNA interference defines a new bacterial defense pathway that further illustrates the diverse roles of Ago proteins.

AGING

\section{A radical reporter}

Nature doi:10.1038/nature13012

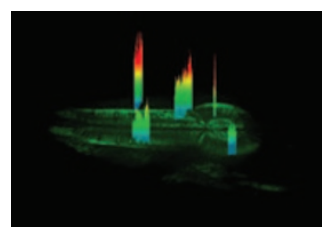

The progressive accumulation of free radicals in the mitochondria due to DNA damage and long-term cellular metabolism has been associated with aging, but the ability to accurately monitor these correlations has been challenging. Recently, a cpYFP reporter (called Mitoflash) was developed to detect random bursts of superoxide production in the mitochondrial matrix, reflecting changes in oxidative stress and metabolism. Shen et al. applied Mitoflash in a model organism of aging, the nematode Caenorhabditis elegans, to determine whether changes in Mitoflash frequency could predict lifespan. They detected two major peaks of Mitoflash activity: an early peak (day 3 ) and a late peak (day 9), when worms begin to die. The authors examined genetic mutants and worms exposed to short- or long-term environmental or pharmacological conditions and surprisingly found a strong negative correlation between the frequency of the early peak and overall worm lifespan. Long-lived worms exhibited lower day-3 Mitoflash frequencies compared to wild-type worms, whereas short-lived worms showed higher frequencies. The authors sought to explain the connection of Mitoflash and lifespan by examining the regulation of mitochondrial metabolic pathways. In particular, upregulation of the glyoxylate cycle in a long-lived mutant promoted metabolic flux through complex II, resulting in decreased superoxide production and Mitoflash activity. The authors caution that the day-3 Mitoflash activity is a marker, not a causal factor of aging. This study suggests that aging is a programmed process in which the mitochondrion organelle has a central role.

GM

\section{LIPID-PROTEIN INTERACTIONS}

\section{It gets specific}

Structure doi:10.1016/j.str.2013.12.018

Membrane phosphoinositides (PIs) are important signaling molecules that can bind selective recognition modules within proteins whose activities are dependent on membrane localization. For instance, $\mathrm{PI}(4) \mathrm{P}$ is found in the eukaryotic Golgi and plasma membrane and is recognized by PH, PX, ENTH and GAT domains within adaptor complexes and lipid-transfer proteins for localization to those membrane subcompartments. DrrA, the Legionella pneumophila guanine nucleotide exchange factor for the Rab1 GTPase, co-opts vesicular traffic within host cells by using its unique PI(4)-binding module (P4M). To understand the basis for binding and exploitation of host processes by DrrA, Del Campo et al. used biochemistry and also solved the crystal structure of a DrrA segment containing the P4M domain with dibutyl PI(4). They found that among seven PIs, DrrA preferentially incorporated into liposomes containing PI(4)P. $K_{\mathrm{D}}$ measurements indicated that both the head group and the diacylglycerol moiety of $\mathrm{PI}(4) \mathrm{P}$ and protein-lipid interactions contribute to the affinity of the P4M for membranes. The PI(4)-bound structure helped explain the PI(4)P specificity, revealing a constricted and deep binding pocket, in which the 4-phosphate is coordinated by two basic and three polar residues. The authors could also predict a DrrA membrane insertion motif (MIM). Mutational analysis validated residues important for binding affinity, particularly ones involved in contacting the PI(4) head group, as well as the location of the PI(4)Pspecific MIM. Although some parallels exist to eukaryotic systems and other PI-binding modules-for instance, with similarities between the DrrA MIM and PX elements found in eukaryotic proteins - these results provide new insights into the mechanisms of $\mathrm{PI}(4) \mathrm{P}$ binding. $M B$

\section{BIOMIMETIC SYNTHESIS}

\section{Sewing up sorbiterrin}

J. Am. Chem. Soc. doi:10.1021/ja500854q

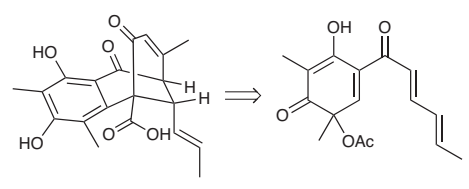

Sorbiterrin A is a newly discovered member of the sorbicillin family of polyketides in which the unusual [3.3.1] ring system was proposed to be generated via an intramolecular aldol/dehydration reaction. Qi et al. now use this proposal to inspire a biomimetic synthesis of this compound. The authors drew on past syntheses of related molecules to design a consecutive Michael addition strategy to construct the quaternary carbon neighboring the aromatic ring, though unstable precursors and challenges in assigning stereochemistry of key spiro intermediates created initial difficulties in the planned approach. Though the authors cleverly used the different photocycloaddition reactivity of the spiro isomers to determine relative stereochemistry, further epimerization of the next, ring-opened intermediates stymied this solution. Treatment of the ring-opened intermediates using silicasupported silver nanoparticles successfully generated the aldol/dehydration product, which was followed by a final saponification to yield sorbiterrin A and its epimer. NMR and X-ray analysis of the saponified compounds confirmed the proposed structure of the natural product. The authors also investigated the role of the silver nanoparticles in promoting the aldol condensation, observing the formation of a radical species that supported two possible mechanistic explanations. This study provides a concise route to an intriguing natural product.

Written by Mirella Bucci, Joshua M. Finkelstein, Catherine Goodman, Grant Miura and Terry L. Sheppard 